\title{
Evaluation of pressure effects on an acoustic thermometer with a single waveguide
}

\author{
Rok Tavčar ${ }^{1}$, Dušan Agrežํㅜ ${ }^{1}$ Samo Beguš ${ }^{1}$ \\ ${ }^{1}$ University of Ljubljana, Faculty of Electrical Engineering, Laboratory of Metrology and Quality, Ljubljana, Slovenia
}

\section{ABSTRACT}

This paper presents the implementation and testing of an acoustic thermometer with a single waveguide. The proposed design has several specific advantages compared to other acoustic designs with waveguides, such as its smaller size, improved sensitivity, and the same common sound path. This paper describes each advantage and the tests used to determine their usefulness. It also shows how to deal with the disadvantages of this design, such as its weak return signals and possible coincidences concerning the return signals. The instrument was calibrated at ice point and then tested in an oil bath from $-30^{\circ} \mathrm{C}$ to $120^{\circ} \mathrm{C}$. In steady state, it had a standard deviation of $0.050{ }^{\circ} \mathrm{C}$, with 66 independent readings per second. The stability of the bath was $10 \mathrm{mK}$ and the uncertainty of the reference thermometer was $0.003^{\circ} \mathrm{C}$.

\section{Section: RESEARCH PAPER}

Keywords: sound; temperature; acoustic; PAT

Citation: Rok Tavčar, Dušan Agrež, Samo Beguš, Evaluation of pressure effects on an acoustic thermometer with a single waveguide, Acta IMEKO, vol. 7, no. 4, article 8, December 2018, identifier: IMEKO-ACTA-07 (2018)-04-08

Editor: Alexandru Salceanu, "Gheorghe Asachi" Technical University of lasi, Romania

Received March 29, 2018; In final form May 31, 2018; Published December 2018

Copyright: (C) 2018 IMEKO. This is an open-access article distributed under the terms of the Creative Commons Attribution 3.0 License, which permits unrestricted use, distribution, and reproduction in any medium, provided the original author and source are credited

Corresponding author: Rok Tavčar, e-mail: Rok.Tavcar@fe.uni-lj.si

\section{INTRODUCTION}

Acoustic thermometers are better known as primary thermometers [1] and have a very high level of accuracy. However, they are not particularly useful for practical measurements because they are too cumbersome. Several proposals have been put forward with the aim of constructing similar thermometers that would be similarly accurate but more practical for everyday use. Examples of designs of practical acoustic thermometers include the twin-tube design [2 - 4] or the use of a measured medium as a waveguide [5 - 6]. Acoustic thermometers with waveguides are useful in harsh environments, such as nuclear reactors [7] or other environments that could influence the measurements, such as a high magnetic field. They are also useful when it is necessary to measure the average temperature of a large volume, such as a chemical reactor. They also benefit from low drift and inexpensive maintenance, as only the gas inside must be replaced to restore operation.

Besides the twin-tube design, which is the most intuitive, there are other designs, such as the joined twin-tube design and single-tube waveguide. The motivation behind exploration of the the single-tube waveguide design is that other designs of acoustic thermometer have inherent flaws that are hard to overcome. For example, the early twin-tube design designed by the authors had fluctuations in readings of approximately $0.5{ }^{\circ} \mathrm{C}$ at room temperature, depending on environmental influences, such as room temperature. This occurred despite the good thermal connection between both tubes, where copper wire was wound around both tubes at the common sound path. In the case of the joined twin-tube design, it was difficult to construct it in such a way that sound split in both tubes evenly. Another motivation is that this design is compact and has a small measurement end, as it is coiled into a helix as opposed to being bent in the form of the letter U. Our proposed design has several advantages compared to other designs, such as its smaller size, improves sensitivity, and the same common sound path. Unfortunately, this design also has some disadvantages, such as the low amplitude of the return signal due to reflection and possible coincidences of the return signals. Section 3 explains how to mitigate these limitations.

The heart of the problem with acoustical thermometers is determining the speed of sound. There are two main approaches to this process: measuring acoustic resonance [8 - 9] or measuring time delay [10 - 11]. This design measures a time delay, more precisely, the time delay between the first and second reflections of soundwaves in the tube. 


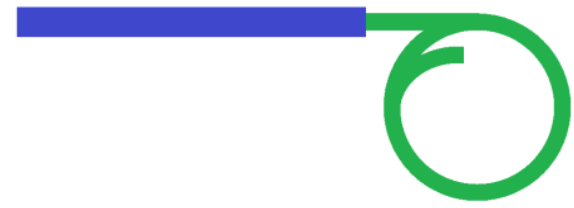

Figure 1. Schematic diagram of the acoustic thermometer with a single waveguide.

\section{ACOUSTIC THERMOMETER}

The scheme of the proposed design is shown in Figure 1. The blue colour indicates the common sound path, which consists of a tube with a larger inner diameter. The green colour indicates the measurement end, a tube with a smaller inner diameter. This tube is coiled into a helix with the smallest bending radius possible for the given tube material. A microphone is placed at the left end of the blue tube. At the junction at which tubes with a different inner radius meet, the first sound reflection is generated, and the second reflection is generated from the end point of the measuring end, where the tube is closed.

\subsection{Speed of sound}

The speed of sound in a medium is mostly influenced by the medium itself and its temperature [12-15]. The relationship is expressed as:

$c_{S}=\sqrt{\frac{T \gamma R}{\bar{m}}}$,

where $T$ is the temperature in Kelvin, $R$ is the gas constant, $\gamma$ is the ratio of specific heats $C p / C v$, and $\bar{m}$ is the molar mass.

Equation 1 is valid in the free field, while in the narrow waveguide, there is an additional dependency on thermal and viscous boundary layers [13], which is a factor at low frequencies (below $5 \mathrm{kHz}$ ).

\subsection{Calculating temperature}

In the proposed design, the speed of sound is calculated using the time of flight method:

$c_{s}=\frac{2 l}{\tau}$,

where $l$ is the length of the tube with the smaller inner radius and $\tau$ is measured transit time. The factor 2 is necessary, as reflected soundwaves travel the same path twice. When inserted into Equation 1, it is transformed to:

$T=\frac{\bar{m}(2 l)^{2}}{\gamma R} \cdot \frac{1}{\tau^{2}}$.

Equation 3 assumes that the first part is constant during temperature measurements, which is valid because the instrument has a slight overpressure in the waveguide compared to the outside atmospheric pressure. The only parameter that changes is the length of the tube. Changes in length are compensated for by the available thermal linear expansion data for stainless steel (AISI 304). The constant in Equation 3 is determined by calibration at an ice point. This is necessary because measurement of the length between the microphone and the sound reflective surfaces is too difficult due to the curvature of the tube.

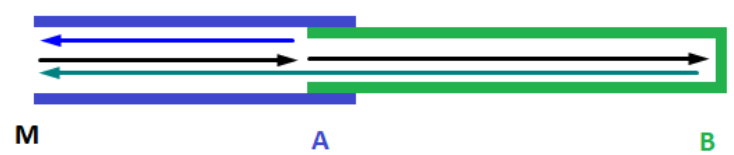

Figure 2. Sound path inside the tubes. $M$ marks the position of a microphone, A marks the position of the first reflection, and B marks the position of the second reflection.

\subsection{Reflections in tubes}

The positions of the microphone and the reflections are indicated in Figure 2. Only reflections occurring due to the change in the inner radius and those at the end of tube are considered. Echoes through metal are not considered, as the speed of sound in steel is more than 10 times faster than in gas. Because of this high difference in speed, most of the soundwaves reflect at the boundary between a gas and a metal.

\subsection{Measuring the transit time}

In this design, the microphone has the dual role of both microphone and speaker. The microphone used in the experiment is a Brüel \& Kjær type 4189 [16] with a custom preamplifier that allows for its use as a speaker. A soundcard EMU-0404 was used to generate and capture electric signals. The microphone was positioned on the tube end (the left side of the blue tube in Figure 1 and Figure 2) with the diaphragm parallel to the soundwaves. The microphone was secured inside the tube with Teflon sealing tape. The transit time measurement was commenced by generating a signal on a computer. The soundcard generated an electric signal that was transmitted by a microphone operating in a speaker mode. The microphone then switched to microphone mode to receive the reflected sound signals. The received signal then travelled to a microphone preamplifier and the soundcard. Thereafter, the microphone switched back to speaker mode. The computer started another measurement after it calculated the transit time.

The excitation signal was a linear sweep, with a frequency range from $5 \mathrm{kHz}$ to $15 \mathrm{kHz}$. Both boundaries were determined in order to ensure the best possible results, since low frequency soundwaves have a different speed and high-frequency soundwaves have a higher attenuation when traveling through a tube. The signal duration was limited by the length of the smaller tube, around $2.5 \mathrm{~ms}$ in this design.

The pulse compression technique and matched filter were used to improve the signal-to-noise ratio (SNR) and to determine the transition time. Because the soundcard sampling frequency was $192 \mathrm{kHz}$, the received signals were up-sampled by a ratio of 10 , and quadratic fit was used in searching for the peaks in the results of the matched filter. Thus, approximately $100 \mathrm{~ns}$ of time delay resolution could be secured.

\section{RELEVANT STUDIES}

Other attempts [1 - 3], [17] at the construction of acoustic thermometers with waveguides have given consistent results over a wide range of temperatures and designs. The results show that the uncertainty and deviation arising in the model described in Equation 3 increased along with a temperature increase. Results in the range of $30{ }^{\circ} \mathrm{C}$ to $50{ }^{\circ} \mathrm{C}$ were a $0.08{ }^{\circ} \mathrm{C}$ deviation from the model and uncertainty amounted to $0.005{ }^{\circ} \mathrm{C}$. In the range up to $600^{\circ} \mathrm{C}$, the deviation from the model increased to 6 ${ }^{\circ} \mathrm{C}$ and the uncertainty to $0.5{ }^{\circ} \mathrm{C}$. In the range from room temperature to $1000^{\circ} \mathrm{C}$, the deviation from the model increased 


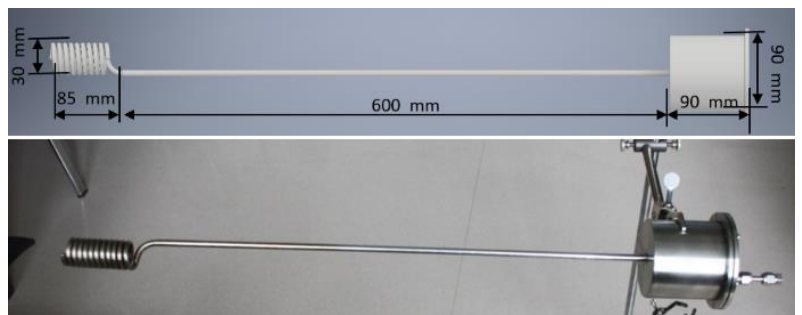

Figure 3. An image of the thermometer with its dimensions, created using CAD (top). A photograph of the finished thermometer (bottom).

to $13{ }^{\circ} \mathrm{C}$ and the uncertainty to $1^{\circ} \mathrm{C}$. Results exceeding $1000{ }^{\circ} \mathrm{C}$ were somewhat different across the designs, but all indicated an increasing deviation from the model along with an increase in temperature.

\section{INSTRUMENT DESIGN}

The instrument was constructed from two main mechanical parts. The first is the measuring part, shown in Figure 3 on the left-hand side in both images.

The basic design consisted of a tube bent into a helix, with one end closed and the other end straightened out and left open. The open end was connected to the second part with another tube (shown in blue in Figure 1 and Figure 2), which had a larger inner and outer diameter than the first part. The second part was cylindrical, housing the microphone and its preamplifier. This part also held the electric and the pneumatic connection to the outside. Inside the helix, the tube and microphone case were filled with air with slight overpressure to minimize the intrusion of the surrounding air.

The DC power supply, soundcard, amplifier, and control electronics were separated from the microphone box. The calculations and data storage were maintained on a PC.

\subsection{Processing the received signal}

The received signal was filtered and synchronized with the transmitted signal. Thereafter, the matched filter model was applied. The matched filter model used was the same as the transmitted signal, which consisted of a linear sine sweep from 5 $\mathrm{kHz}$ to $15 \mathrm{kHz}$, and was additionally filtered by the inverse frequency filter before matching. The parameters of the inverse frequency filter were handpicked for ensuring the best performance.

\subsection{The lengths of the tubes and their ratios}

As mentioned above, one major problem with this design is coincidence of the return signals. The proposed solution is to carefully choose the dimensions of the tubes and the ratios between them. In Table 1 and Table 2, the calculations for two optimal options are displayed. Length 1 is the length of the straight tube in Figure 1, and Length 2 is the length of the helix. Table 1 and Table 2 show the sound path lengths for two different tube length ratios.

Figure 2 shows the sound paths in the tubes, with the length ratios calculated as in Table 2. The sound started at $\mathrm{M}$ and travelled to point A. Here, the path split, and half of the sound

Table 1. Calculations for sound paths with length ratio 1:0.5.

\begin{tabular}{|l|l|c|}
\cline { 2 - 3 } \multicolumn{1}{c|}{} & Length 1=1 $\mathrm{m}$ & Length 2=0.5 m \\
\hline First return & $2 \mathrm{~m}$ & $3 \mathrm{~m}$ \\
\hline Second return & $4 \mathrm{~m}$ & $6 \mathrm{~m}$ \\
\hline Third return & $6 \mathrm{~m}$ & $9 \mathrm{~m}$ \\
\hline
\end{tabular}

Table 2. Calculations for sound paths with length ratio 1:1.5.

\begin{tabular}{|l|c|c|}
\cline { 2 - 3 } \multicolumn{1}{c|}{} & Length $1=1 \mathrm{~m}$ & Length $2=1.5 \mathrm{~m}$ \\
\hline First return & $2 \mathrm{~m}$ & $5 \mathrm{~m}$ \\
\hline Second return & $4 \mathrm{~m}$ & $10 \mathrm{~m}$ \\
\hline Third return & $6 \mathrm{~m}$ & $15 \mathrm{~m}$ \\
\hline
\end{tabular}

was reflected back to $\mathrm{M}$ (blue arrow), and half travelled further to point $\mathrm{B}$. At point $\mathrm{B}$, all sound was reflected back to M.

In this case, the first reflected signal was from the first reflection, and the second from the second reflection.

In the second case, the first reflected signal was from the first reflection, the second was the second return from the first reflection, and the third was from the second reflection.

Both cases were chosen so that the signal return from the second reflection was between two returns from the first reflection to minimize the influence between both reflections. For this instrument, it was better to choose the second ratio (with the longer measuring end) to ensure improved sensitivity. Improved sensitivity at the longer measurement end was a consequence of the measurement change in the time of flight for the transmitted signals. The longer measurement end delays the returned signal more so than the short one. The total length was limited by the attenuation of the returned signals, and this depended on the radii of the tubes, the microphone, and the frequency of the used sound waves. The preliminary tests showed that the maximum length of the tubes and microphone was $1.5 \mathrm{~m}$, i.e., $0.6 \mathrm{~m}$ for the common path and $0.9 \mathrm{~m}$ for the measuring end.

\section{EXPERIMENTAL SETUP}

Before the main test could be performed, a few other preliminary tests that were not temperature-related were required to be performed to determine the thermometer parameters, such as microphone SNR and the effect of switching the microphone preamplifier between the microphone and the speaker mode. The main test consists of two parts. The first is the thermometer calibration at an ice point to determine Equation 3's parameters. The second part is the actual temperature test in the oil bath: from $-30{ }^{\circ} \mathrm{C}$ to $120^{\circ} \mathrm{C}$. The bath used had a temperature stability of $10 \mathrm{mK}$, and the reference thermometer was $0.003{ }^{\circ} \mathrm{C}$.

One test cycle included 16 uniformly spaced steady states, each two hours long. In total, there were four cycles, which resulted in one week of testing. This test showed the standard deviation and matching with the model. It also showed the presence of any hysteresis or drift.

After the main test was complete, three additional tests for measuring acoustic SNR versus pressure were undertaken, increasing the temperature range and gas mixing time. The test setup for measuring the acoustic SNR and gas mixing time was similar to the ice point calibration setup. The test included maintaining the measuring end at a constant temperature $\left(0^{\circ} \mathrm{C}\right)$ and changing the pressure in the tube. To measure usability at a higher temperature, the thermometer was put in an oven with a controlled temperature.

\section{EXPERIMENTAL RESULTS}

In Figure 4, the readings from the reference thermometer versus the acoustic thermometer are plotted. The line is almost linear, with a few bumps. These bumps were consequences of the larger time constant of the reference thermometer compared to the acoustic thermometer, and they were only present in the transient states. 


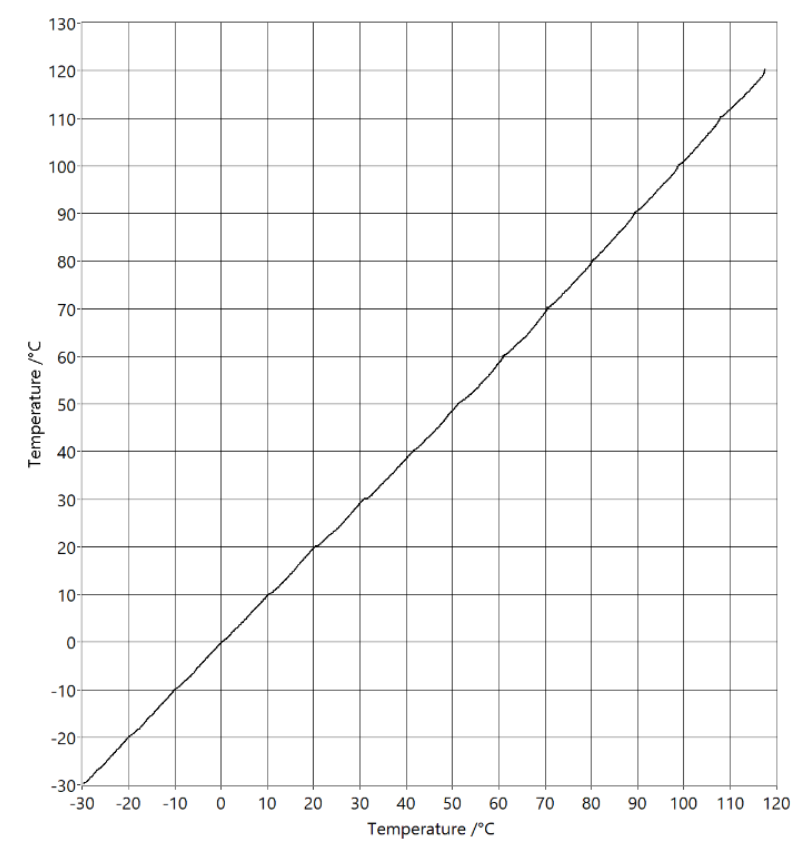

Figure 4. A plot of the reference thermometer versus the acoustic thermometer temperature readings.

Considering Figure 4 and Figure 5, the model used (Equation 3) was good in the temperature range of $-30{ }^{\circ} \mathrm{C}$ to $20^{\circ} \mathrm{C}$, but at higher temperatures, there was a deviation that increased with the temperature. Further analysis showed that above $60{ }^{\circ} \mathrm{C}$, the deviations from the model linearly increased along with the temperature increase, with a slope of $0.05{ }^{\circ} \mathrm{C} /{ }^{\circ} \mathrm{C}$.

Figure 5 shows the temperature error of the acoustic thermometer versus the measured temperature compared to the reference thermometer. The deviations from the model above 60 ${ }^{\circ} \mathrm{C}$ are clearly shown.

Figure 6 shows a small part of the measurements around $0{ }^{\circ} \mathrm{C}$. The readings from the reference thermometer are marked in red, and the readings from the acoustic thermometer are marked in black. Readings from the acoustic thermometer were averaged to better show the drift in the figures. The time at $0 \mathrm{~s}$ was the start of the experiments.

The steady state shown in Figure 6 was 3.1 days after calibration. It shows that the thermometer did not have a longterm drift, but it did have some short-term drift.

Figure 7 shows a small part of the readings around $100{ }^{\circ} \mathrm{C}$. The difference compared to the previous figure is that the scale for the acoustic thermometer is on the right-hand side of the graph. The difference between the scales is $1.8^{\circ} \mathrm{C}$. It is presented in this manner for ease of comparison.

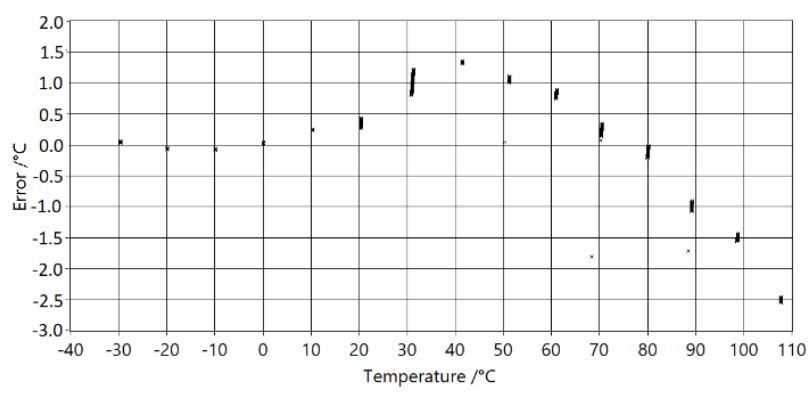

Figure 5. Temperature error versus acoustic thermometer temperature readings.

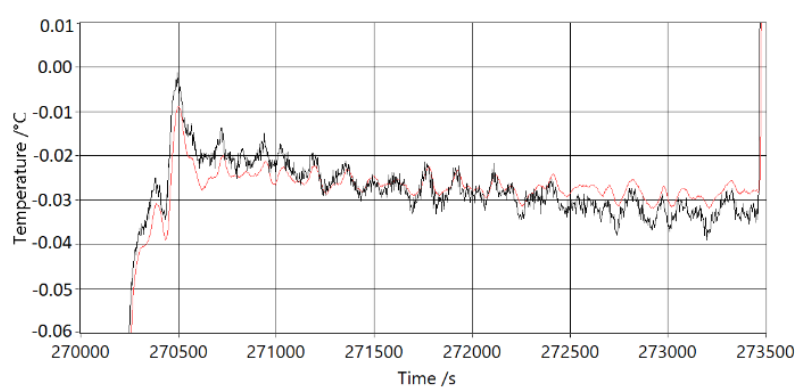

Figure 6. Small part of the steady state around $0{ }^{\circ} \mathrm{C}$.

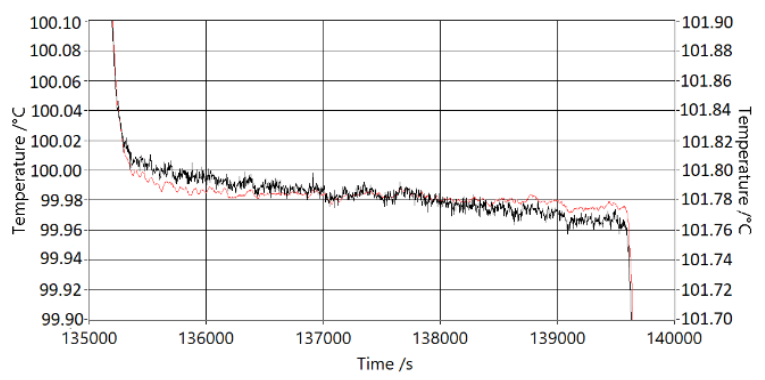

Figure 7. Small part of the steady state around $100{ }^{\circ} \mathrm{C}$.

Figure 7 shows that at high temperatures, a similar short-term drift occurred as was seen for lower temperatures. Furthermore, at these higher temperatures, the model deviated from the measured results.

The SNR tests showed that the signal duration of $2.5 \mathrm{~ms}$ was enough in order to ensure sufficient SNR after the pulse compression for temperature measurements. Switching between microphone and speaker mode did produce spikes in the returned signal, but they were harmless due to a long pause between one mode and another, and they did not influence the peak position after cross-correlation.

Heating the housing (but not the measuring end) with a heat gun did not cause any temperature error, but it did produce noise in the measurements while a heat gun was turned on.

\subsection{Length of tubes}

The lengths and ratios of the tubes were selected before the production of the thermometer and were based on a prototype without overpressure. Because of the effects of overpressure on acoustic SNR (details described in section 8), the selected lengths were not ideal. The results of the selected lengths included an overlap in the return signal starting from $200-300{ }^{\circ} \mathrm{C}$ (depending on the temperature in the common sound path). Because of the increased acoustic SNR, the length of the transmitted signal can be shorter, which directly influences the lengths of the tubes.

As signal overlap happens only when the temperature in the measuring end is too high compared to the common sound path, it is possible to raise the temperature limit higher by heating the common sound path. This did not influence how the temperature was calculated in this experiment because the temperature in the common sound path does not have a direct influence on the measured temperature. This method can practically raise the temperature range by $100{ }^{\circ} \mathrm{C}$, as it is limited by a maximum permissible working temperature of the microphone and electronic components used.

Figure 8 shows the problem that limits high-temperature operations. At $4 \mathrm{~ms}$, the first return from the change in radius started, as shown in blue in Figure 2. At $8 \mathrm{~ms}$, the second return from the change in radius started, and at $9 \mathrm{~ms}$ the reflection from 


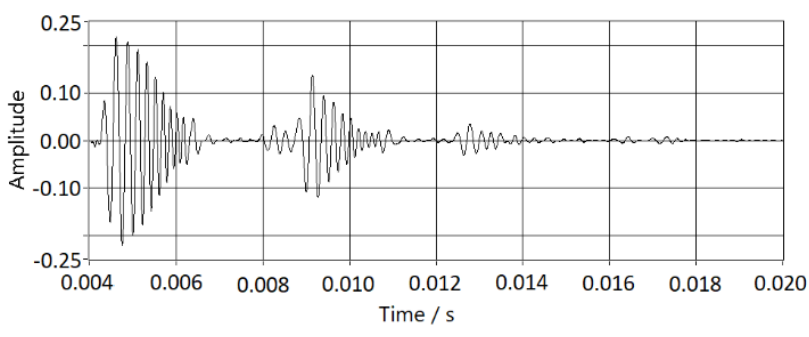

Figure 8. Received acoustic signal at $400^{\circ} \mathrm{C}$.

the end of the measurement end started, as shown in green in Figure 2. The reflection from the end of the measurement end was faster than the second reflection from the change in the inner radius return. This created a coincidence in the acoustic signal and distorted the temperature measurements.

\subsection{Revised calculation of tube lengths for high temperatures}

While the ratio calculations described in section 4.2 are important for a design, they do not consider changes in temperature between a common sound path and measurement end. The temperature in a common sound path has a greater influence on the delay and amplitude of reflected signals than is estimated using our thermometer [18]. This simplifies tube length calculations, because the main focus is reducing the length of the common sound path as much as possible. The lowest length is limited by the signal duration and expected temperatures in a common sound path.

\section{GAS MIXING IN TUBES}

We conclude that the drift occurred due to the mixing of air with pure gas (argon), as shown in Figure 6 and Figure 7. The duration of this process was long because the gas exchange in the thermometer was only accomplished by changing pressure in the tube without creating a vacuum in it. Gas mixing in a tube is a slow process [19], having a significant effect on the speed of sound [20]. This process was over two weeks for this thermometer and uncertainty level. In this case, the calibration and tests were undertaken one week after the start of the gas exchange, so gas mixing had not completed finished by this point, resulting in drift. Figure 6 and Figure 7 also show that the temperature readings from the thermometer fell during the steady state, which supports our hypothesis. The speed of sound in argon is lower than in air, so when the gas mix included more argon, the sound needed more time to travel the same distance. This resulted in a lower temperature being calculated using Equation 3.

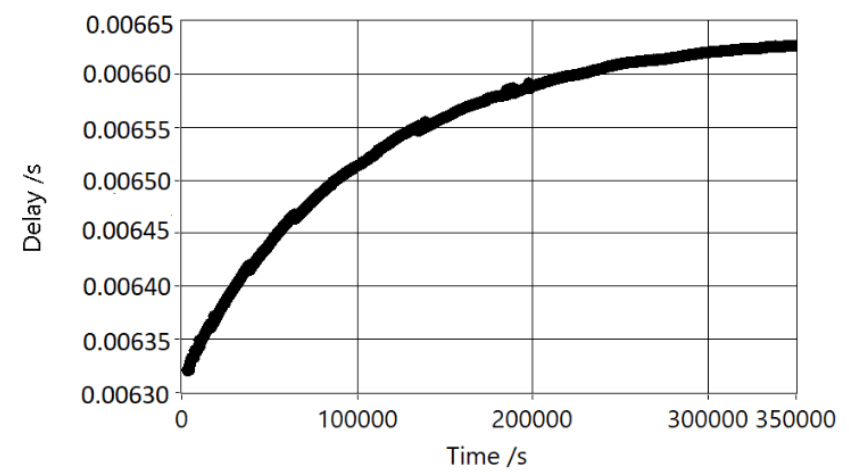

Figure 9. Measuring delay at a constant temperature while the gases inside the thermometer mix.
Figure 9 shows the start of the effect caused by mixing two gases. At this point, the thermometer was filled with air at room pressure, and then it was filled with argon until the pressure inside reached four bars. Thereafter, it was left at a constant temperature and pressure.

\section{ACOUSTIC SNR DEPENDANCE ON PRESSURE}

The SNR of the acoustic signal in the tubes depend on the gas pressure. Figure 10 is a graph of the measured SNR of the acoustic signal from the thermometer versus the pressure of the gas inside of the thermometer.

The acoustic SNR increased along with an increase in pressure, or attenuation in the tube was falling. This occurred because of the reduced absorption of the medium, which is inversely proportional to the square root of pressure. This has both good and bad implications for temperature measurements. Temperature measurements with a higher acoustic SNR have a smaller standard deviation, but the measurement frequency has to be reduced because acoustic signals need more time to settle down after each measurement with lower attenuation. This is because additional reflections need to be attenuated to minimize the impact on the next measurement.

Figure 11 shows the temperature standard deviation versus pressure. Despite the reduced measurement frequency, it was better to increase the pressure in the thermometer than to undertake faster measurements and then average them. The measurement frequency was 66 readings per second at room pressure and 15 readings per second at 4 bars.

The standard deviation was much smaller than in the bath test because this test was done in a less noisy environment than the bath, which had some noisy moving parts. The other reason is that the bath had a stability of $10 \mathrm{mK}$.

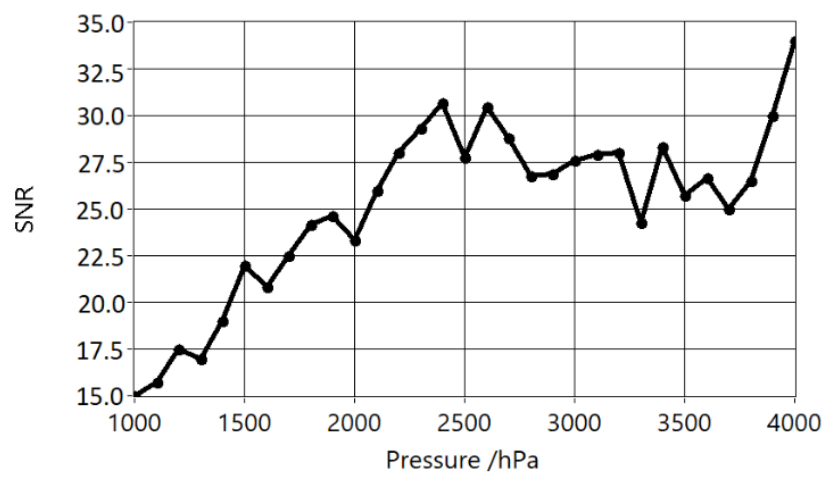

Figure 10. Signal-to-noise ratio of the acoustic signal versus the pressure inside the thermometer.

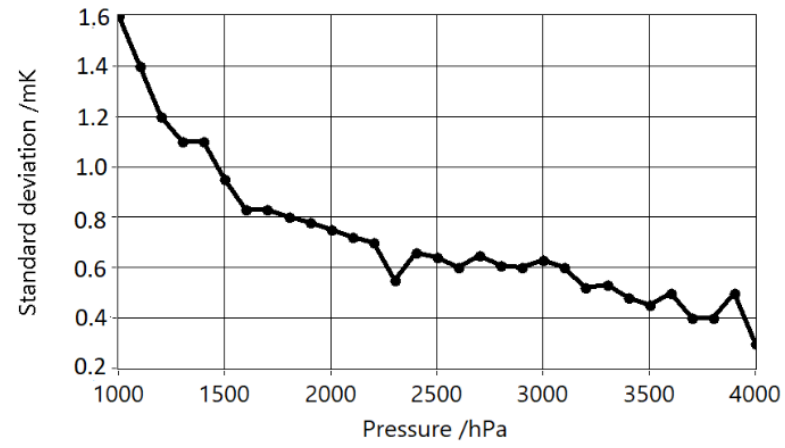

Figure 11. Temperature standard deviation at ice point versus pressure inside the thermometer. 


\section{CONCLUSION}

The design proposed in this work offers new advantages compared with similar acoustic thermometers, while also retaining their benefits. The key change and advantage of this design is a compact coiled measuring end, since it enables this design to have very high sensitivity with a small size. Other important changes include the use of sound frequencies at which sound speed is less dependent on frequency. Furthermore, the dual use of a microphone as a speaker improves the mechanical stability of the system. The experimental results showed two problems with the current implementation. The first is the gas mixing in the tubes, particularly its timescale. The second is the deviation from the model used. Gas mixing can be accelerated by using a vacuum pump, and once the process is complete, it does not have an influence until the gas composition changes again. After mitigating the deviation from the model, the calculated standard deviation on 66 samples (1 second of measurement) at $100{ }^{\circ} \mathrm{C}$ was $70 \mathrm{mK}$ with the oil bath running and $30 \mathrm{mK}$ with the oil bath powered down. It was also shown that with a higher gas pressure in the waveguide, it is possible to reduce the temperature standard deviation if the measurements are slowed down to compensate for the reduced attenuation of sound in the tubes. The range of the thermometer can be increased to $300{ }^{\circ} \mathrm{C}$ without changes in the design or gas used. $\mathrm{By}$ heating the common sound path, the range can be increased to $400{ }^{\circ} \mathrm{C}$.

Using the described algorithms and tube designs, it is possible to achieve a temperature error of less than $2{ }^{\circ} \mathrm{C}$ temperature error in the $-30{ }^{\circ} \mathrm{C}$ to $120{ }^{\circ} \mathrm{C}$ range with a calibration at one temperature. With a calibration at more reference temperatures or by applying the correction to the measured temperature, it is possible to achieve a temperature error below $0.4{ }^{\circ} \mathrm{C}$ at $120^{\circ} \mathrm{C}$.

The tests showed three areas in which improvements are necessary: using a speaker with a higher SPL, improved acoustic insulation between the outside and inside of the housing, and filling the acoustic tube with a pure gas, e.g., argon or nitrogen, to eliminate drift due to gas mixing. Another possibility is to use xenon gas, which has a much lower speed of sound: $178 \mathrm{~m} / \mathrm{s}$ compared to $319 \mathrm{~m} / \mathrm{s}$ for argon and $349 \mathrm{~m} / \mathrm{s}$ for nitrogen, at a gas temperature of $20^{\circ} \mathrm{C}$.

Increasing the sound pressure level and improving the acoustic insulation would reduce the standard deviation of the measurements and susceptibility to outside noise. A greater SNR can also be achieved by a higher gas pressure and improved acoustic insulation. Changing the gas inside of the housing and tubes would also reduce the short-term drift and deviation from the model. Using xenon would also increase the sensitivity by almost factor of two compared to nitrogen or air.

The problem of acoustic signal coincidence can be solved by heating a common sound path or using gas with lower speed of sound. The first method is limited by a microphone's tolerance to temperature, as it is at one end of a common sound path. The second method requires finding a usable gas with a lower speed of sound. Another solution to this problem is to use acoustic noise cancellation techniques to cancel or reduce the amplitude of the second return from the first reflection, thus eliminating or reducing acoustic signal coincidence.

\section{REFERENCES}

[1] B. Fellmuth, C. Gaiser, J. Fischer, Determination of the Boltzmann constant - status and prospects, Meas. Sci. Technol. 17 (2006), pp. 145-160.

[2] H. J. Aulfes, Akustisches gasthermometer mit dunnen sensorrohren, PhD Dissertation, University of Paderborn, 1993

[3] M. de Podesta, G. Sutton, R. Underwood, S. Legg, A. Steinitz, Practical acoustic thermometry with acoustic waveguides, Int. J. Thermophys 31 (2010), pp. 1554-1566.

[4] N. Amir et al., "Inspecting U-tube bundles using acoustic pulse reflectometry", Proc. of the Asme Power Conf., Jul. 21-23, 2009, Albuquerque, New Mexico, USA, pp. 141-145.

[5] R. Underwood, T. Gardiner, A. Finlayson, J. Few, J. Wilkinson, S. Bell, J. Merrison, J. J. Iverson, M. de Podesta, A combined noncontact acoustic thermometer and infrared hygrometer for atmospheric measurements, Meteorol. Appl. 22 (2015), pp. 830 835.

[6] R. Underwood, T. Gardiner, A. Finlayson, S. Bell, M. de Podesta, An improved non-contact thermometer and hygrometer with rapid response, Metrologia 54 (2017), pp 9-15.

[7] J. B. Roes, D. L. Peat, The development of an acoustical thermometer for a graphite matrix nuclear fuel element, IEEE Transactions on Nuclear Science 14 (1967), pp. 348-359.

[8] A. Elliott, Passive acoustic thermometry, Proc. of the Internoise 2016, Aug. 21-24 2016, Hamburg, Germany, pp. 622-629.

[9] J. H. Apfel, Acoustic thermometry, Rev. Sci. Instrum. 33 (2004), pp. 428-430.

[10] L. C. Lynnworth and E. H. Carnevale, "Ultrasonic thermometry using pulse technique", in: Temperature: Its Measurement and Control in Science and Industry, Volume 4. Instrument Society of America (editor), 1972, pp. 715-732.

[11] X. B. Mi, S. Y. Zhang, J.J. Zhang, and Y. T. Yang, "Automatic Ultrasonic Thermometry", Journal of Nanjing University 39 (2003), pp. 517-523.

[12] J. W. S. Rayleigh, Theory of Sound, Vol II, Sects. 255-266, Macmillan, London, 1894.

[13] L. E. Kinsler, A. R. Frey, A. B. Coppens, and J. V. Sanders (editors), Fundamentals of Acoustics, Vol. xii Wiley, New York, U.S.A., 2000 , p. 548.

[14] H. Tijdeman, On the propagation of sound waves in cylindrical tubes, J. Sound Vib. 39 (1975), pp. 1-33.

[15] T. Yazaki, Y. Tashiro, T. Biwa, "Measurements of sound propagation in narrow tubes", Proc. R. Soc. A 463 (2007), pp. 2855-1862.

[16] https://www.bksv.com/en/products/transducers/acoustic/micr ophones/microphone-cartridges/4189.

[17] G. Sutton, M. de Podesta, R. I. Veltcheva, P. Gélat, H. D. Minh, G. Edwards, Twin-tube practical acoustic thermometry: theory and measurements up to $1000^{\circ} \mathrm{C}$, Meas. Sci. Technol. 26 (2015), pp 1-15.

[18] R. Tavcar, D. Agrez, S. Begus, "Acoustic thermometer with single waveguide", Proc. of the 22nd IMEKO TC4 International Symposium \& 20th International Workshop on ADC Modelling and Testing: Supporting World Development through Electrical and Electronic Measurements, Sept. 14-15, 2017, Iasi, Romania, pp. 244-248.

[19] Photoacoustic, Photothermal and Photochemical Processes in Gases. Ed. P. Hess, Springer-Verlag, Berlin a.o.1989. pp. 166

[20] D. A. Bohn, Environmental Effects on the Speed of Sound,J. Audio Eng. Soc. 36 (1988), pp. 223-231. 